\title{
Nanoscale Stripe Arrays Templated on Moiré Patterns in Graphite
}

\author{
Michael J. McNally, Mumin M. Koc, Gauthier Torricelli, Klaus von Haeften \\ Department of Physics and Astronomy, University of Leicester, University Road, Leicester, \\ UK, LE1 $7 R H$
}

\begin{abstract}
Large areas of nanoscale stripe arrays were produced by drop casting silica nanoparticle solutions on highly oriented pyrolytic graphite surfaces at room temperature and imaged with atomic force microscopy. The alignment of the striped areas always reflected the threefold symmetry of the graphite surface. Two different patterns were observed, with different coverages, line separations and mutual orientation, being offset by $30^{\circ}$. Measurement of the relative angles and separations of the line patterns showed a very good match with an underlying Moiré pattern, resulting from the rotation of the top graphene layers. Closer-spaced lines were attributed to the zig-zag direction of the Moiré pattern whereas wider-spaced lines belonged to the armchair direction. The different abundance and apparent difference in long-term stability suggested that stability was governed by the number of reactive vertices per unit area as opposed to the number of vertices per line-length. While sequential images recorded over several days revealed long term stability of all zig-zag arrays, attachment and detachment of single nanoparticles was observed. By contrast, arrays aligned in the armchair direction appeared and vanished collectively, suggesting condensation and evaporation of a fluid of nanoparticles floating on the surface.
\end{abstract}

Keywords: nanoparticles, HOPG, graphene, self assembled monolayers, moiré

\section{Introduction}

Self assembled monolayers (SAMs) provide a method for bottom up assembly of nanostructured surfaces. The ability to control surface geometry and chemistry has led to hydrophobic coatings [1], electro-chemical [2] and bio5 chemical sensors [3]. Applications are emerging for capturing biomolecules and nanoparticles [4] and as elements in electronic devices [4, 5. The physics of nanostructuring on highly oriented pyrolytic graphite (HOPG) is of relevance to graphene; SAMs on graphene have been used to provide n-doping [6], develop

\footnotetext{
Email addresses: mjm48@le.ac.uk (Michael J. McNally), kvh6@le.ac.uk (Klaus von Haeften)
}

Preprint submitted to Surface Science

September 4, 2015 
high sensitivity detectors for biomolecules [5] and enable atomic layer deposition

10 of metal oxides onto graphene sheets [7. Linear arrays on surfaces have been investigated for the control of atomic and molecular transport [8], to produce surfaces with directional conductivity $[9$ and to modify the optical properties of the surface [10].

HOPG is widely used as a substrate as it can be easily cleaved to provide

15 an atomically flat substrate and is relatively chemically inert. It does not readily form the covalent bonds typical for SAMs on metal or semiconductor surfaces [4. Consequently self-assembly is dominated by the intermolecular forces between adsorbents. Adsorbate-Substrate interactions are chiefly limited to van der Waals interactions [11 or stacking $\pi$ - $\pi$ bonds in cyclic aromatic hydrocar20 bons [4, 11. However, edge and point defects in HOPG show comparatively higher reactivities and are preferential sites for adsorption. Superlattices in HOPG can be seen of a long range, well ordered 'defect' state, which modifies the local density of states and reactivities of the surface. It has been shown that superlattices can modify the density, packing and alignment of SAMs [12.

25 Superlattices are regularly observed in HOPG and form due to interference (Moiré) patterns formed when the top sheet of graphene is rotated with respect to the underlying crystal [13, 14]. The same Moiré patterns are observed in epitactic graphene on Ruthenium and Iridium substrates. All of these systems have been used to template the growth or adsorption of metal nanoparticles [15,

so 16, 17, 18, 19, 20, 21] and C60 22 in two dimensional hexagonal arrays. These interactions should also influence the growth of arrays of particles which tend to form linear, one dimensional structures.

Here we report the observation of linear arrays of weakly interacting particles on HOPG, produced by dropcasting dilute nanoparticle solutions onto freshly 35 cleaved surfaces. We demonstrate that they follow an underlying Moiré pattern. The different directions along the Moiré pattern are shown to have different reactivities. Furthermore we introduce a simple model to explain observed angular misalignment and to suggest expressions for array sizes and defect densities.

\section{Methods}

40 2.1. Nanocluster Synthesis

The nanoparticle solutions used in this work were produced by mixing water and silicon atoms. A silicon cluster source deposited onto a liquid nitrogen cooled target. Simultaneously, a spray of water vapour was deposited onto the cold target. This method has been fully described in previous publica-

45 tions [23, 24]. The resulting solution was found to contain a stable phase of silicon nanoparticles with a narrow size distribution centred at $1.3 \mathrm{~nm}$. Analysis of this size distribution revealed that the nanoparticles had been produced entirely from the silicon atoms accompanying the cluster beam [25]. The produced particles showed stable luminescence over a period of 3 months [23], 50 further measurements showing that fluorescence remains stable over years, indicating that the particles are chemically stable and have weak inter-particle reactions. 


\subsection{Nanoparticle film growth}

Nanoparticle films were produced on highly oriented pyrolytic graphite (HOPG).

${ }_{55}$ Films were produced by drop-casting of nanoparticle solution onto a freshly cleaved HOPG surface in ambient atmosphere. Successive dilutions of nanoparticle solutions with milli-Q purified water demonstrated that the film coverage was correlated with the dilution factor. A dilution factor of between 10 and 20 to one was determined to be appropriate to produce monolayer nanopartiso cle films from a $5 \mu \mathrm{L}$ droplet. Height measurements of these films were then used to determine nanoparticle diameters [26. For further production of films it was decided to dilute the samples with isopropanol, to facilitate wetting of the surface and speed the drying process.

Samples imaged in this work were nanoparticles in water, diluted 10:1 with

65 isopropanol. Both laboratory wash quality isopropanol and ultra pure HPLC grade isopropanol (Sigma-Aldrich) were used for dilution without significantly changing the results. To prepare a nanoparticle film for imaging in the AFM, a $5 \mu \mathrm{L}$ droplet of 10:1 isopropanol to nanoparticle solution was pipetted onto a freshly cleaved HOPG wafer $\left(0.8^{\circ}\right.$ minimum spread, Pi Kem $)$ under a laminar

70 flow hood. The drop rapidly dried and the wafer was then introduced to the vacuum load lock, and from there to the analysis chamber, maintained at a pressure below $10^{-9}$ mbar. In order to disentangle solution and substrate effects, the same nanoparticle solution was used to prepare samples on different HOPG wafers, as well as repeated cleaving of the same crystal.

75 2.3. Atomic Force Microscopy

The atomic force microscope (AFM) used was a variable temperature AFM from Omicron Nanotechnolgy. This was operated in non-contact, constant force mode, all measurements were taken at room temperature. A $20 \mathrm{~nm}$ radius SiN tip was used (Mikromasch) with a resonance frequency of $307 \mathrm{kHz}$ and a so nominal stiffness of $40 \mathrm{Nm}^{-1}$. The vertical axis was calibrated on single and double step edges of the graphite, of $3.5 \AA$ and $7 \AA$ height respectively. Series measurements were made over 24 hours. Each image in the series was one of four images acquired from different scanning directions. Two complete scans were made, top to bottom and bottom to top, each generating two images of left to right and right to left scanning directions. These scans were recorded continuously at 27 minutes per complete scan. All images are selected to have the same scanning directions, series images are therefore separated by 54 minute intervals. All images were analysed using the software packages WSXM [27], Gwyddion [28] and ImageJ [29].

\section{3. Results and Discussion}

\subsection{Stripe Domains}

Drop cast films of highly diluted samples on HOPG wafers were investigated with an AFM under ultra-high vacuum (UHV) conditions. Samples showed regions of bare HOPG having a height distribution FWHM of $0.6 \AA$, which we 
take as the error in the $\mathrm{Z}$ accuracy of measurements. The heights of the features are proportional to the atomic force and therefore not necessarily proportional to the true heights. When different materials are present on the surface the interaction changes which will inevitably lead to the reading of a different 'height' of the AFM in the constant height mode. Also we assume that the laterally unresolved, relative coverage of material under the tip is reflected in the apparent measured height. Regions of discrete layer films were observed: for areas of complete coverage, the height distribution showed distinct peaks for a first and second layer coverage, reflecting the formation of stacked monolayers. Step heights ranged from 1.3-1.5 nm. In areas of lower density stripe arrays can be observed on the surface, as shown in figure 1. These stripes have heights ranging from 4-6 A. Stripe domains are not observed in control samples of either water only, isopropanol only, or a mixture. Furthermore, when the nanoparticle solution is not diluted with isopropanol, stripe domains are not observed.

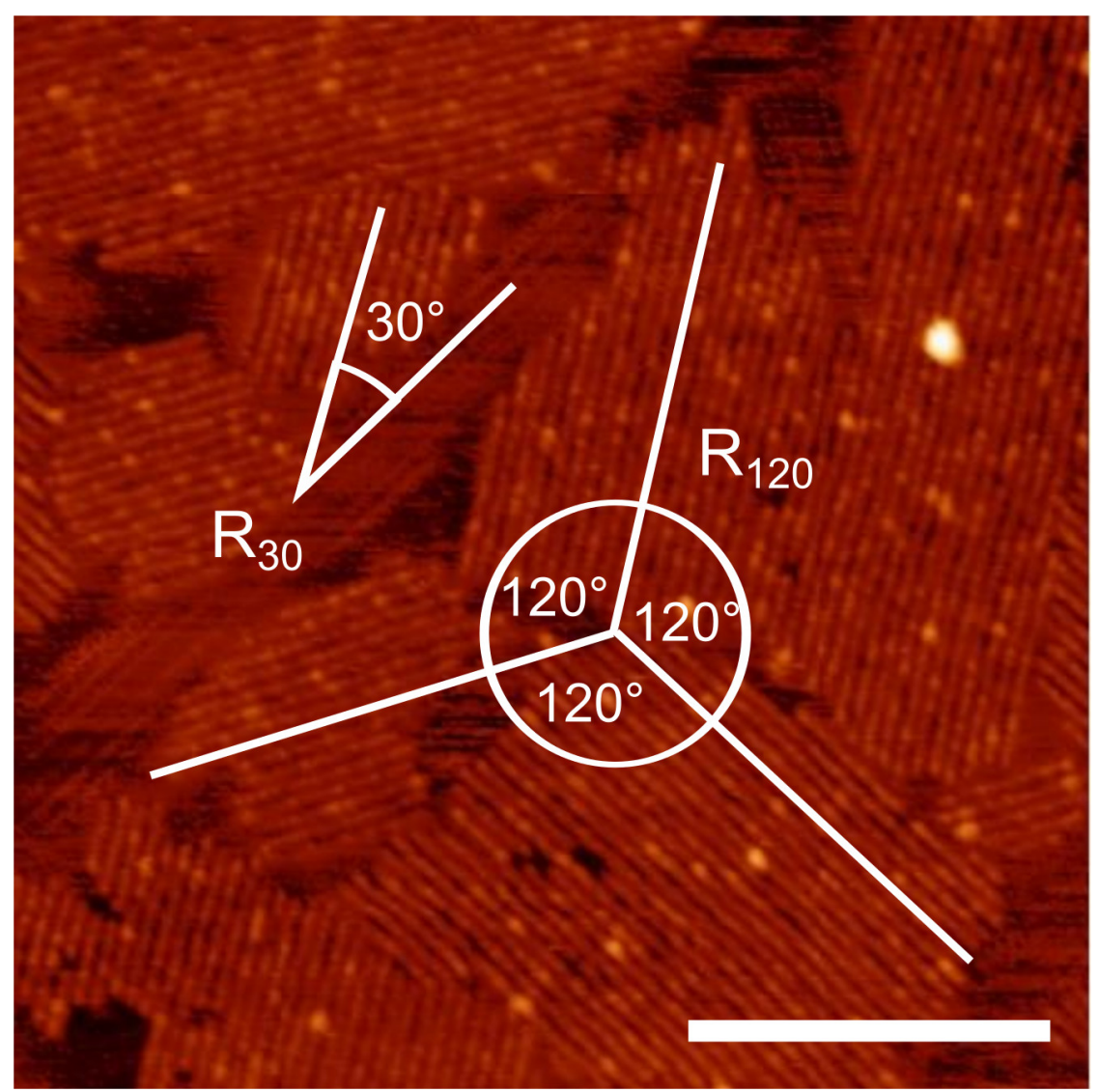

Figure 1: Region of aligned domains. Domains are labelled to represent their relative orientation, $R_{120}$ represents the predominant arrays consistently forming at $120^{\circ}$ to each other, whilst $R_{30}$ represents the comparatively less frequent array aligned at $30^{\circ}$ to $R_{120}$. The scale bar is $100 \mathrm{~nm}$. 
The aligned domains covering the majority of the surface show a threefold

\subsection{Moiré Templating}

We will now show that the predominant force driving alignment on the surface is the interaction between adsorbed particles and the substrate. Specifically, 


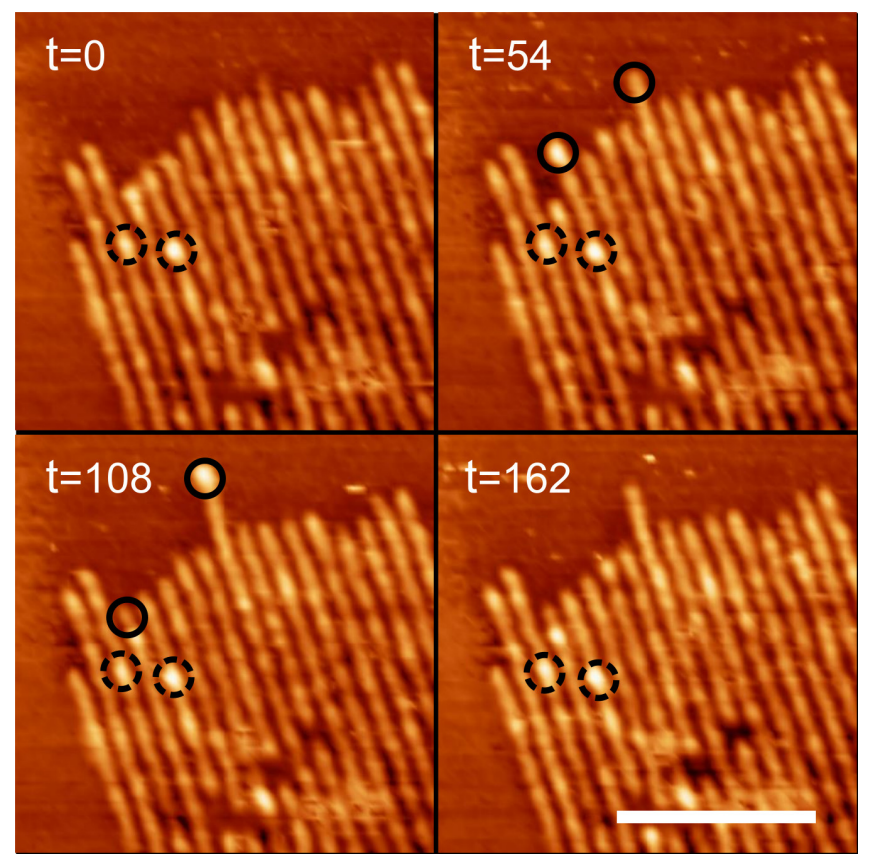

Figure 2: Series of images from a set of 24 images, showing array growth and dissolution proceeding via discrete steps. Dashed circles: These points are unchanged over all 20 images. Solid circles: Discrete attachments since the previous frame. Time is in minutes. The scale bar is $50 \mathrm{~nm}$.

growth is governed by interactions with the Moiré pattern formed by rotation of the top sheet of graphene with respect to the bulk HOPG. When two honeycomb 155 lattices are overlayed with a mutual rotation, as in figure 3 , the interference or Moiré pattern generates a honeycomb superlattice. The lattice constant $\mathrm{a}_{M}$ of a Moiré pattern generated from two stacked sheets of graphene (lattice constant $\mathrm{a}_{g}=1.42 \AA$ ) rotated by an angle of $\theta$ is given by equation 1$][13$.

$$
a_{M}=\frac{a_{g}}{2 \sin \frac{\theta}{2}}
$$

Rotational defects are commonly observed in the stacking of graphene planes in both HOPG [13, 30, 14, 31 and multi-layer graphene [32, 33, and lead to the formation of hexagonal superlattices. The generation of these defects has been ascribed to the cleaving process for HOPG [33, or by treatment of HOPG with chlorinated hydrocarbons [14, 30]. The superlattice patterns are visible in scanning tunnelling microscope measurements, as regions within the Moiré 165 pattern have different local densities of states (LDOS) at the fermi level [13, 14. In the sheet of graphene forming the top layer of an HOPG wafer, it is possible to identify two distinct triangular sub-lattices. In the A lattice, each carbon atom sits directly above another in the sheet below, in an $\alpha$ site. The $\mathbf{B}$ lattice is made up of carbon atoms sitting directly above a vacancy in the lattice below, 


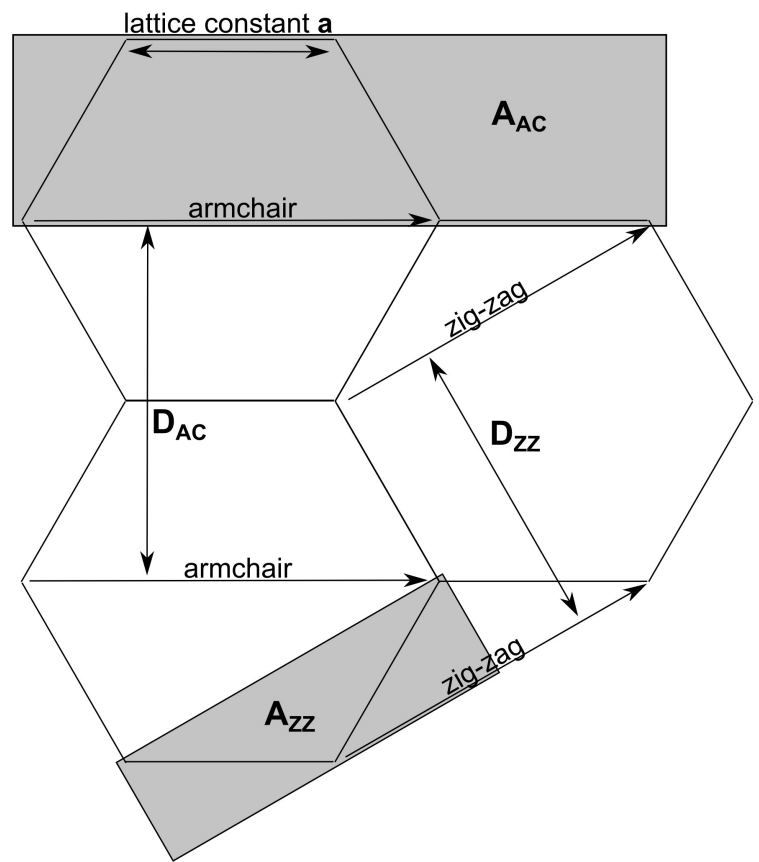

Figure 3: Diagram of a hexagonal lattice showing the zig-zag and armchair directions, the separations between lines following these directions, $\mathrm{D}_{Z Z}$ and $\mathrm{D}_{A C}$ and the lattice constant a.

170 a $\beta$ site 14. In a Moiré pattern formed between the top layer of HOPG and the bulk, as illustrated in figure 4 , within the new superlattice, there are sites, M- $\beta$, with higher densities of exposed $\beta$ sites. Preferential adsorption of single atoms and molecules onto $\beta$ sites has been experimentally observed [34, 35. Larger clusters and nanoparticles selectively adsorb on the M- $\beta$ sites due to their higher 175 density of $\beta$ sites [13].

The predominance of $\mathrm{R}_{120}$ arrays indicates that there is a preferred direction for alignment. A simple explanation for this is the difference between the zig-zag and armchair directions illustrated in figure 3. If we assume that the vertices in the diagram represent reactive sites, comparing the densities of vertices in each direction will indicate the preferred direction. There are two ways of measuring the densities - the density per unit length, $\rho X$, measured along the direction of alignment, or the density per unit area, $\rho A$, where areas are bounding rectangles along the alignment direction. The ratio of linear densities is: $\frac{\rho X_{Z Z}}{\rho X_{A C}}=\frac{\sqrt{3}}{2}$, with the armchair direction having higher density. The ratio of densities per unit 185 area is $\frac{\rho A_{Z Z}}{\rho A_{A C}}=2$. Here the zig-zag direction has higher density. In both cases, there is a preferred direction, armchair has higher linear density, whilst zig-zag has higher area density.

To determine the preferred direction we observe, we have to compare the 


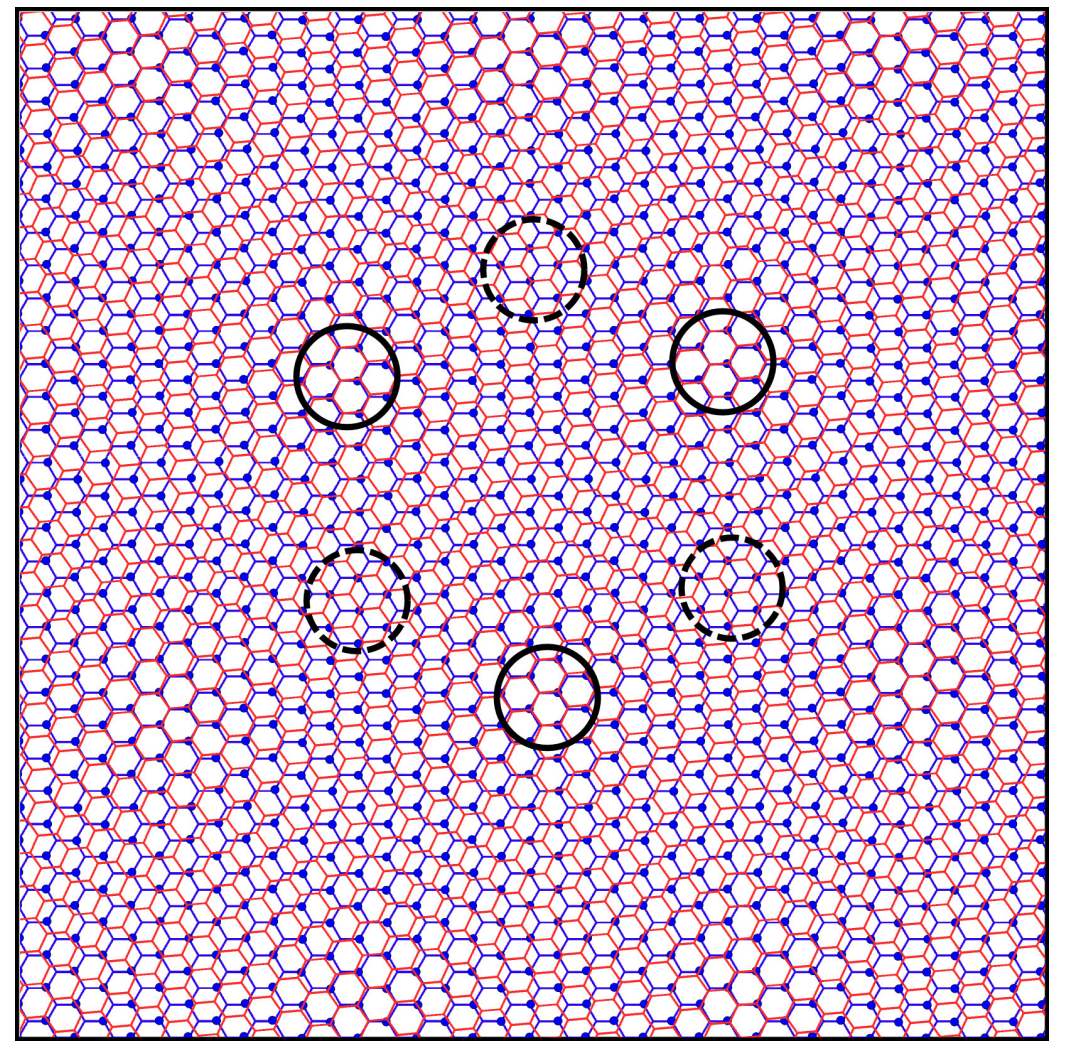

Figure 4: Illustration of sites in HOPG superlattice formed by rotating the top sheet (red) by $5^{\circ}$ relative to the lower layer (blue). Adapted from [13. $\alpha$ sites in the bulk lattice, which overlap atoms in the previous layer, are marked with a blue circle. $\beta$ sites, where the carbon atom in the current layer does not overlap an atom in the previous, have a higher density of states and are the typical sites for adsorption of molecular species. The solid black circles show the area in the Moiré pattern with the fewest exposed $\beta$ sites. The dashed circles enclose the areas with the highest density of exposed $\beta$ sites.

separations of lines in the arrays. As (from figure 3 p $\mathrm{D}_{A C}$ is greater than $\mathrm{D}_{Z Z}$, 190 with the largest separation belong to the to figure 1, the separation of $R_{120}$ lines is smaller than $R_{30}$ lines. This shows that the preferred alignment direction is in the zig-zag direction. This also shows that the number of reactive sites per unit area is more important than per unit length. Comparing the values for separations in the $\mathrm{R}_{120}$ arrays, 5.5 $195 \pm 0.2 \mathrm{~nm}$, with the separation of the lines in the $R_{30}$ array, $6.2 \pm 0.4 \mathrm{~nm}$, we find that the ratio is roughly $\frac{\sqrt{3}}{2}$. This is the ratio of the lengths $\mathrm{D}_{Z Z}$ and $\mathrm{D}_{A C}$ from figure 3 the ratio of distances between lines in the zig-zag and armchair direction. This further verifies that the $\mathrm{R}_{120}$ arrays are following a zig-zag and the $R_{30}$ arrays are following an armchair in an underlying Moiré pattern. 


\subsection{Zig-Zag and Armchair growth}

At moderate coverage, arrays form spaced apart on the substrate. This is illustrated in figure 5, a series of eight images from a set of 24 acquired continuously over 24 hours. Two arrays follow the $\mathrm{R}_{120}$ alignment, despite the separation between them. A third $R_{30}$ aligned array grows between them. Starting as a faint set of lines, the $R_{30}$, it grows to meet the $R_{120}$ aligned array (top left), the ends of the lines of both arrays meeting precisely. This further corroborates that the ordering interaction arises from the substrate, rather than from adsorbents having a hexagonal shape.

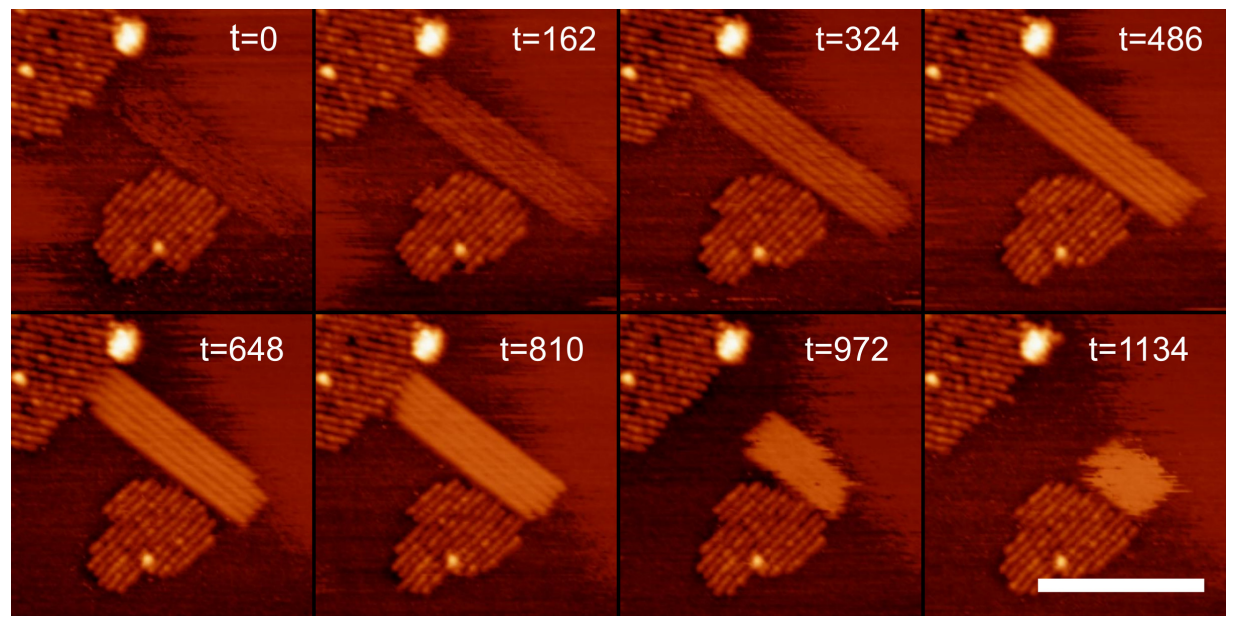

Figure 5: Time series from 24 images, showing growth and dissolution of an aligned region at 30 degree alignment to neighbouring islands. The central region is already present in the first image as an array with low density and coverage, which then grows in height and length. It begins to dissipate from the top left, reducing substantially in length but not reducing height. In the final frames, it has become unstable over the timescale of a single line scan (roughly 4 seconds) which could be interpreted as melting or evaporation of the array. Time is in minutes. The scale bar is $100 \mathrm{~nm}$.

The $R_{30}$ array starts as a faint set of lines, which then increases slightly in length, but rapidly in measured height (atomic force). It then shrinks significantly in length, but without a corresponding reduction in height. In the final images this array has become unstable, changing position over the duration of a single line scan (roughly 4 seconds). Over the time period of the whole series, 21 the $\mathrm{R}_{120}$ arrays are largely unchanged apart from a few attachments along their edges. This clearly shows a substantial difference in stability between the $\mathrm{R}_{120}$ and $\mathrm{R}_{30}$ directions. Consequently, this reveals that the armchair direction is less reactive and forms weaker bonds with adsorbates than the zig-zag direction.

Whilst it is clear that linear arrays of adsorbents can develop on Moiré patterns, and will preferentially follow zig-zag directions, our observations cannot clarify how the arrays start growing. We can suggest two possible models. In one model, a single particle adsorbs to a $\beta$ site, either in the Moiré or the top 
two layers of HOPG, and the array grows from this point by piecewise growth as successive particles arrive. Particles do not 'see' the Moiré, but instead simply find the lowest energy position in which they can align. From figure 4 we can see that this will tend to be along the zig-zag direction of the Moiré. This model is supported by the observations of single attachment events in figure 2 .

In another model, an ensemble of particles freezes or condenses spontaneously into an array. The whole array will then have stability dependent on the

230 interaction between the particles which make it up, and the interaction between the array and the underlying Moiré in HOPG. Arrays which cover the most M- $\alpha$ sites in the Moiré pattern will have the lowest stability, and this will apply to arrays in the armchair direction, or to out of alignment directions. The rapid growth and dissolution of the $R_{30}$ array in figure 5 , particularly the apparent simultaneous growth of the entire array would appear to support this model.

Some attachment and detachment events, such as those observed in figure 2. may be induced or mediated by tip interactions. However, we note that in figure 5 there are changes in the total coverage of the surface with both $\mathrm{R}_{120}$ and $\mathrm{R}_{30}$ arrays, and hence in the total number of particles present. Particles must 240 diffuse in and out of the imaged areas over the duration. This indicates that tip interaction may drive array growth through transfer of energy and momentum rather than by carrying individual nanoparticles.

It is likely that both particle by particle and spontaneous growth play a role in array formation, with different contribution dependent on the properties of 245 the absorbent and the direction of growth along the Moiré pattern. In particular, it seems that growth along the zig-zag direction proceeds by successive addition of new particles, whilst the weaker forces in the armchair direction facilitate cooperative condensation and melting.

\subsection{Angular Defects}

250 Some indicators towards the dynamics of array growth can be seen in figure 6. This is an enlargement of figure 1. This figure demonstrates that there are slight deviations from the ideal $120^{\circ}$ of $\mathrm{R}_{120}$ or the $30^{\circ}$ of $\mathrm{R}_{30}$ when comparing angles between arrays. These angular deviations can also be observed as defects within the arrays themselves, as shown in the large array across the top of figure

255 6. Such angular defects in the arrays could arise from arrays being able to follow either the underlying Moiré or one of the top two layers in the graphite. In this case, arrays following the top layer of graphene would grow until they reach an M- $\alpha$ site in the Moiré pattern, where it would become strongly favourable to change direction and grow along either the Moiré orientation, or the second layer orientation.

Array angles were measured in over 20 images, all recorded from a single sample prepared on one HOPG wafer, including images recorded in different scan directions. These were converted into an absolute angular deviation from the nearest $R_{120}$ or $R_{30}$ rotation, with one array in each image defined as having 265 a deviation of $0^{\circ}$ to use as a reference point. The distribution of these angles is shown in figure 7. The Gaussian fit shown by the black line has a peak at $2.2^{\circ}$. From surface diffraction studies of HOPG and multi-layer graphene on $\mathrm{SiC}, \pm$ 


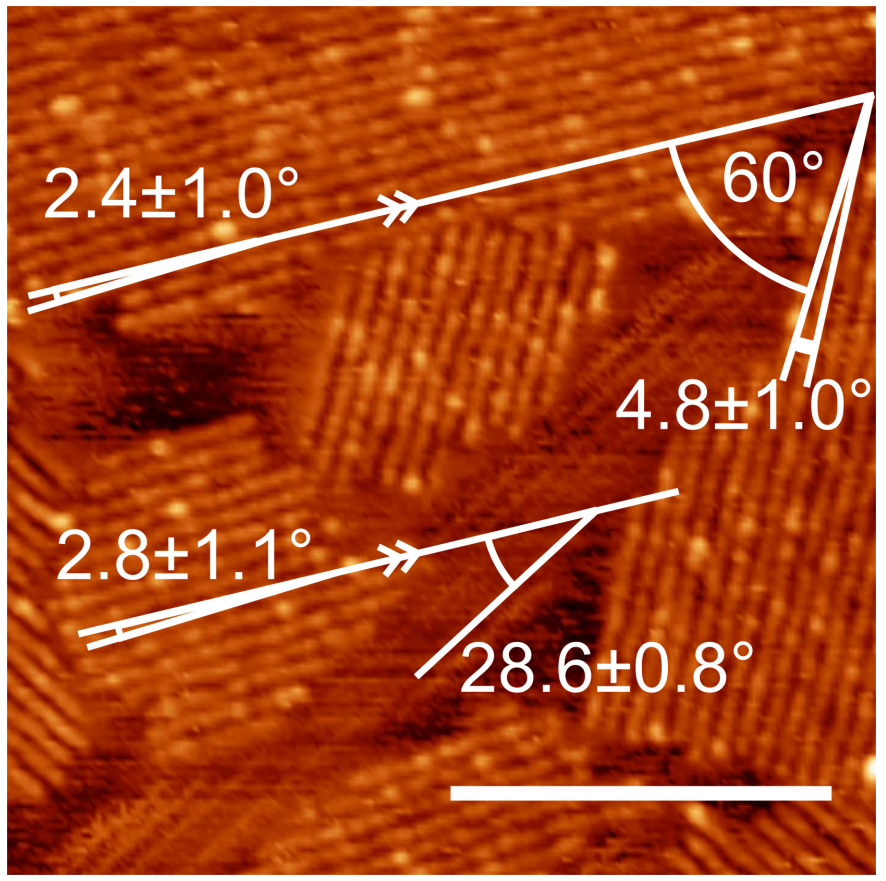

Figure 6: Measurement of the relative angles of stripe domains from figure 1. Angles are measured from the local Fast Fourier Transforms (FFT) of stripe arrays. The scale bar is 100 nm.

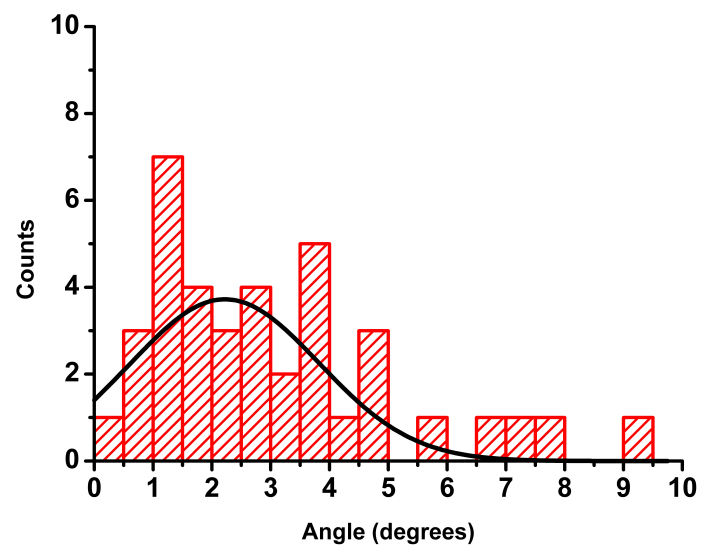

Figure 7: Distribution of angles between arrays expected angular separation $\left(120^{\circ}, 30^{\circ}\right)$ and measured values. Histogram: Values were measured from both FFT of selected regions within images, and using angular measurement tools. Line: Gaussian fit to the data, centre $=2.2^{\circ}$, $\mathrm{FWHM}=3.8^{\circ}$

$2.204^{\circ}$ is known to be the most common angular mismatch between layers [33. We can use equation 1 to calculate a value for the separation of lines for this 
Moiré angle, which returns values of $5.5 \mathrm{~nm}$ for zig-zag and $6.4 \mathrm{~nm}$ for armchair lines, in good agreement with our measured values.

If we follow the assumption that these angular mismatches arise from growth along the top, second and Moiré layers, we can calculate the average distance any line can grow before it must reach an $\mathrm{M}-\alpha$ site. In the case of following

275 the Moiré pattern exactly, the line can continue indefinitely, until encountering some other defect or adsorbent on the HOPG surface. However, if following one of the top two layers, lines will eventually reach defects after an average distance $L_{Z Z D}=\frac{a_{M}}{2 \sin \left(\frac{\theta}{2}\right)}$, the diagonal across a zig-zag in the Moiré pattern at the angle $\pm \frac{\theta}{2}$ between the Moiré pattern and the top and second layer in the HOPG. Combined with equation 1 this gives a characteristic length between defects of:

$$
L_{Z Z D}=\frac{a_{g}}{4 \sin ^{2}\left(\frac{\theta}{2}\right)}
$$

Which, for $\theta=2.2^{\circ}$ gives a value $\mathrm{L}_{Z Z D}=96 \mathrm{~nm}$. This is consistent with the size of arrays and the frequency of defects we see in figure 1. where the complete coverage ensures that the array sizes are not governed by the number of adsorbents available for growth.

\section{Conclusion}

In this work we have produced well ordered linear arrays by drop casting solutions of silica nanoparticles, isopropanol and water onto HOPG. We have observed two distinct patterns of array, $\mathrm{R}_{120}$ and $\mathrm{R}_{30}$, with the same threefold symmetry, but with different line separations and mutually rotated by $30^{\circ}$. The zag and armchair directions on an underlying Moiré pattern. We have identified that the $\mathrm{R}_{120}$ arrays follow the zig-zag direction in the Moiré pattern and the $\mathrm{R}_{30}$ arrays follow the armchair direction. The greater abundance and apparent longterm stability of the $\mathrm{R}_{120}$ arrays indicates that the zig-zag direction is favourable unit area, whilst the armchair direction has a greater number of vertices per unit length. Hence we conclude that the number of reactive vertices per unit area is determining stability.

All $R_{120}$ arrays were relatively stable over the duration of the experiment (greater than one day at room temperature). We have observed individual attachment and detachment of particles from the tips of $\mathrm{R}_{120}$ arrays. Particles of different measured heights attach in the same positions in the arrays, indicating that the particles forming the arrays are inhomogeneous in size and reactivity. Growth by attachment and detachment of single particles has only been observed in the case of the zig-zag aligned $\mathrm{R}_{120}$ arrays. $\mathrm{R}_{30}$ arrays were observed less frequently, and were not observed exhibiting the same discrete attachment and detachment events. An $\mathrm{R}_{30}$ array was observed growing and dissipating, whilst nearby $\mathrm{R}_{120}$ arrays exhibited minimal changes. Moreover, the $\mathrm{R}_{30}$ array appeared spontaneously and grew in intensity homogeneously along it's length, 

resembling evaporation, further suggesting collective action. The difference in lifetime between arrays provides further evidence for the greater stability in arrays growing in the zig-zag direction. Furthermore, our observations indicate that the dynamics of array growth depend on the direction.

A consequence of the Moiré pattern is that directions are only well defined above a minimum length. Below this critical length, particles growing in linear arrays (which could be oriented in any direction) will grow until they reach an unfavourable location in the Moiré pattern. At this point they then realign towards the nearest Moiré direction. We observe a number of small angular context of this model. These small angular deviations are distributed around the rotation angle of $2.2^{\circ}$, the most commonly observed rotation angle between successive sheets of HOPG. This angle corresponds to the observed separation of lines in the pattern. Furthermore, we have derived a simple expression which determines the average length before reaching a defect.

Existing observations of the organisation of nanoparticles into well ordered, 2D arrays on HOPG or graphene Moiré shows that inorganic nanoparticles will preferentially adsorb onto specific sites within the Moiré pattern. In our system we produce nanoparticles by directly mixing silicon atoms with water molecules.

330 Production in water, as well as carefully controlling drying conditions by dilution with isopropanol, was key to producing linear aligned arrays. However the underlying force driving linear growth is still unknown and requires further investigation.

We would expect to see similar linear arrays for other small inorganic nanoparticles where a small molecular group can provide the potential for linear growth, whilst a larger inorganic core 'sees' the Moiré potential. Templating onto a Moiré pattern offers a method to generate and orient aligned structures of inorganic nanoparticles. Moiré patterns with specific spacings are available by growing epitaxial graphene on different substrates, for example Ru or Ir, enabling the 340 rational design of monolayers with controlled spacings and orientations for future applications or analytical techniques. The method outlined in this paper could also be applied to templating on few or bilayer graphene for optical or electronic applications.

\section{References}

[1] A. Ulman, Formation and Structure of Self-Assembled Monolayers, Chem. Rev. 96 (4) (1996) 1533-1554. doi:10.1021/cr9502357.

[2] J. J. Gooding, F. Mearns, W. Yang, J. Liu, Self-Assembled Monolayers into the 21st Century: Recent Advances and Applications, Electroanalysis 15 (2) (2003) 81-96. doi:10.1002/elan.200390017.

350

[3] D. Samanta, A. Sarkar, Immobilization of bio-macromolecules on selfassembled monolayers: methods and sensor applications., Chem. Soc. Rev. 40 (5) (2011) 2567-2592. doi:10.1039/c0cs00056f. 
[4] S. A. Claridge, W.-S. Liao, J. C. Thomas, Y. Zhao, H. H. Cao, S. Cheunkar, A. C. Serino, A. M. Andrews, P. S. Weiss, From the bottom up: dimensional control and characterization in molecular monolayers., Chem. Soc. Rev. 42 (7) (2013) 2725-45. doi:10.1039/c2cs35365b.

[5] L. Newton, T. Slater, N. Clark, A. Vijayaraghavan, Self assembled monolayers (SAMs) on metallic surfaces (gold and graphene) for electronic applications, J. Mater. Chem. C. 1 (3) (2013) 376-393. doi:10.1039/c2tc00146b

[6] B. Li, A. V. Klekachev, M. Cantoro, C. Huyghebaert, A. Stesmans, I. Asselberghs, S. De Gendt, S. De Feyter, Toward tunable doping in graphene FETs by molecular self-assembled monolayers., Nanoscale 5 (20) (2013) 9640-4. doi:10.1039/c3nr01255g.

[7] X. Wang, S. M. Tabakman, H. Dai, Atomic layer deposition of metal oxides on pristine and functionalized graphene, J. Am. Chem. Soc. 130 (26) (2008) 8152-8153. arXiv:0805.3712, doi:10.1021/ja8023059.

[8] F. Patolsky, Y. Weizmann, I. Willner, Actin-based metallic nanowires as bio-nanotransporters., Nat. Mater. 3 (10) (2004) 692-695. doi:10.1038/ nmat1205.

[9] R. Silerova, L. Kalvoda, D. Neher, A. Ferencz, J. Wu, G. Wegner, Electrical Conductivity of Highly Organized Langmuir - Blodgett Films of Phthalocyaninato-Polysiloxane, Chem. Mater. 10 (1998) 2284-2292. doi: $10.1021 / \mathrm{cm} 980251 \mathrm{v}$.

[10] W. Luo, W. van der Veer, P. Chu, D. L. Mills, R. M. Penner, J. C. Hemminger, Polarization-Dependent Surface Enhanced Raman Scattering from Silver 1D Nanoparticle Arrays, J. Phys. Chem. C Lett. 112 (31) (2008) 11609-11613. doi:10.1021/jp803455s.

[11] J. Otsuki, STM studies on porphyrins, Coord. Chem. Rev. 254 (19-20) (2010) 2311-2341. doi:10.1016/j.ccr.2009.12.038.

[12] S. B. Son, J. R. Hahn, Effects of the Superlattices on STM Imaging of Self-organized Substituted Alkyl Chain Monolayers on a Graphite Surface, Bull. Korean Chem. Soc 33 (12) (2012) 4155-4160.

[13] J. Xhie, K. Sattler, M. Ge, N. Venkateswaran, Giant and Supergiant Lattices on Graphite, Phys. Rev. B 47 (23) (1993) 15835 - 15841.

[14] Y. Wang, Y. Ye, K. Wu, Simultaneous observation of the triangular and honeycomb structures on highly oriented pyrolytic graphite at room tem-

q perature: An STM study, Surf. Sci. 600 (2006) 729-734. doi:10.1016/j. susc.2005.12.001.

[15] S. Billinge, Nanoparticle structures served up on a tray, Nature 495 (2013) 453. doi:10.1038/495453a. 
[16] K. Donner, P. Jakob, Structural properties and site specific interactions of Pt with the graphene/Ru(0001) moiré overlayer., J. Chem. Phys. 131 (16) (2009) 164701. doi:10.1063/1.3246166.

[17] D. Franz, S. Runte, C. Busse, S. Schumacher, T. Gerber, T. Michely, M. Mantilla, V. Kilic, J. Zegenhagen, A. Stierle, Atomic structure and crystalline order of graphene-supported Ir nanoparticle lattices, Phys. Rev. Lett. 110 (6) (2013) 1-5. doi:10.1103/PhysRevLett.110.065503

[18] Q. Liao, H. J. Zhang, K. Wu, H. Y. Li, S. N. Bao, P. He, Nucleation and growth of monodispersed cobalt nanoclusters on graphene moiré on $\mathrm{Ru}(0001)$., Nanotechnology 22 (12) (2011) 125303. doi:10.1088/ 0957-4484/22/12/125303.

[19] A. T. N'Diaye, S. Bleikamp, P. J. Feibelman, T. Michely, Two-dimensional Ir cluster lattice on a graphene moié on $\operatorname{Ir}(111)$, Phys. Rev. Lett. 97 (21) (2006) 1-4. doi:10.1103/PhysRevLett.97.215501

[20] Y. Pan, M. Gao, L. Huang, F. Liu, H. J. Gao, Directed self-assembly of monodispersed platinum nanoclusters on graphene Moié template, Appl. Phys. Lett. 95 (9) (2009) 1-4. doi:10.1063/1.3223781.

[21] E. Sutter, P. Albrecht, B. Wang, M. L. Bocquet, L. Wu, Y. Zhu, P. Sutter, Arrays of $\mathrm{Ru}$ nanoclusters with narrow size distribution templated by monolayer graphene on Ru, Surf. Sci. 605 (17-18) (2011) 1676-1684. doi:10.1016/j.susc.2011.01.026

[22] J. Lu, P. S. E. Yeo, Y. Zheng, Z. Yang, Q. Bao, C. K. Gan, K. P. Loh, Using the Graphene Moire Pattern for the Trapping of C60 and Homoepitaxy of Graphene, ACS Nano (1) (2011) 944-950. doi:10.1021/nn204536e.

[23] A. Brewer, K. von Haeften, In situ passivation and blue luminescence of silicon clusters using a cluster beam/H2O codeposition production method, Appl. Phys. Lett. 94 (2009) 261102. doi:10.1063/1.3167355.

[24] K. von Haeften, C. Binns, A. Brewer, O. Crisan, P. B. Howes, M. P. Lowe, C. Sibbley-Allen, S. C. Thornton, A novel approach towards the production of luminescent silicon nanoparticles: Sputtering, gas aggregation and co-

(1) deposition with H2O, Eur. Phys. J. D 52 (1-3) (2009) 11-14. doi:10.1140/ epjd/e2009-00024-x.

[25] G. Torricelli, A. Akraiam, K. von Haeften, Size-selecting effect of water on fluorescent silicon clusters., Nanotechnology 22 (31) (2011) 315711. doi: $10.1088 / 0957-4484 / 22 / 31 / 315711$.

[26] G. Galinis, G. Torricelli, A. Akraiam, K. Von Haeften, Measurement of cluster-cluster interaction in liquids by deposition and AFM of silicon clusters onto HOPG surfaces: Height distributions of stacked layers as fingerprints of cluster-cluster interaction in solution, J. Nanoparticle Res. 14 (8) (2012) 1057. doi:10.1007/s11051-012-1057-4. 
[27] I. Horcas, R. Fernández, J. M. Gómez-Rodríguez, J. Colchero, J. GómezHerrero, A. M. Baro, WSXM: A software for scanning probe microscopy and a tool for nanotechnology, Rev. Sci. Instrum. 78 (1) (2007) 013705. doi:10.1063/1.2432410.

[28] D. Nečas, P. Klapetek, Gwyddion: an open-source software for SPM data analysis, Cent. Eur. J. Phys. 10 (1) (2011) 181-188. doi:10.2478/ s11534-011-0096-2.

[29] M. D. Abràmoff, P. J. Magalhães, S. J. Ram, Image processing with imageJ, Biophotonics Int. 11 (7) (2004) 36-41. arXiv:1081-8693, doi:10.1117/ 1.3589100 .

[30] H. S. Wong, C. Durkan, Unraveling the rotational disorder of graphene layers in graphite, Phys. Rev. B - Condens. Matter Mater. Phys. 81 (4) (2010) 1-5. doi:10.1103/PhysRevB.81.045403.

[31] D. L. Miller, K. D. Kubista, G. M. Rutter, M. Ruan, W. A. De Heer, P. N. First, J. A. Stroscio, Structural analysis of multilayer graphene via atomic moiré interferometry, Phys. Rev. B 81 (12) (2010) 1-6. doi:10. 1103/PhysRevB.81.125427.

[32] J. Hass, W. A. de Heer, E. H. Conrad, The growth and morphology of epitaxial multilayer graphene, J. Phys. Condens. Matter 20 (32) (2008) 323202. doi:10.1088/0953-8984/20/32/323202.

[33] L. B. Biedermann, M. L. Bolen, M. A. Capano, D. Zemlyanov, R. G. Reifenberger, Insights into few-layer epitaxial graphene growth on $4 \mathrm{H}-\mathrm{SiC}$ (0001) substrates from STM studies, Phys. Rev. B - Condens. Matter Mater. Phys. 79 (12) (2009) 1-10. doi:10.1103/PhysRevB.79.125411.

[34] E. Ganz, K. Sattler, J. Clarke, Scanning tunneling microscopy of the local atomic structure of two-dimensional gold and silver islands on graphite, Phys. Rev. Lett. 60 (18) (1988) 1856-1859. doi:10.1103/PhysRevLett. 60.1856

[35] U. Muller, K. Sattler, J. Xhie, N. Venkateswaran, J. Raina, Scanning tunneling microscopy of single platinum atoms and small platinum clusters on highly oriented pyrolytic graphite, J. Vac. Sci. Technol. B 9 (2) (1991) 829. doi:10.1116/1.585521. 